\title{
ERROR BOUND ANALYSIS AND SINGULARLY PERTURBED ABEL-VOLTERRA EQUATIONS
}

ANGELINA M. BIJURA

Received 5 May 2003 and in revised form 16 June 2004

Asymptotic solutions of nonlinear singularly perturbed Volterra integral equations with kernels possessing integrable singularity are investigated using singular perturbation methods and the Mellin transform technique. In particular, it is demonstrated that the formal approximation is asymptotically valid.

\section{Introduction}

Singularly perturbed nonlinear Volterra problems with kernels of integrable singularity are investigated. The singularity encountered in these problems is that of a layer type; the solution is valid everywhere except in a thin layer, very near to the origin. This thin layer is called the initial layer, the region of rapid variations or the inner layer region. The region outside the initial layer is the outer region. There are many perturbation problems which may be properly called singular but which are not of the layer type. Examples of such problems are problems of secular type.

The following singularly perturbed nonlinear Volterra integral equation with a weakly singular kernel is particularly considered:

$$
\varepsilon x(t)=g(t)+\frac{1}{\Gamma(\alpha)} \int_{0}^{t} \frac{a(t, s)}{(t-s)^{1-\alpha}} f(x(s), s) d s, \quad 0<\varepsilon \ll 1,0<\alpha<1,0 \leq t \leq T,
$$

where $g, a$, and $f$ are continuous functions. These functions may as well depend on $\varepsilon$, but it is assumed here that they are independent. When $g$ is written as $g(t ; \varepsilon)$, one requires that $\lim _{\varepsilon \rightarrow 0} g(0 ; \varepsilon)=0$. It is known from the standard theory of Volterra integral equations that this problem has a solution $x(t ; \varepsilon)$ in $C[0, T]$ for all $\varepsilon>0$. If one is interested in the solution $x(t ; \varepsilon)$ for small values of $\varepsilon$ and assumes an expansion of the form

$$
x(t ; \varepsilon) \approx \sum_{n=0}^{\infty} \varepsilon^{n} x_{n}(t),
$$

Copyright (C) 2004 Hindawi Publishing Corporation Journal of Applied Mathematics 2004:6 (2004) 479-494 2000 Mathematics Subject Classification: 41A60, 45G05, 26A33 URL: http://dx.doi.org/10.1155/S1110757X04305024 
the leading order term $x_{0}(t)$ obeys

$$
0=g(t)+\frac{1}{\Gamma(\alpha)} \int_{0}^{t} \frac{a(t, s)}{(t-s)^{1-\alpha}} f\left(x_{0}(s), s\right) d s, \quad 0 \leq t \leq T
$$

This is a Volterra integral equation of the first kind. For this equation to have a continuous solution, $g(t)$ cannot be merely continuous, the forcing function must be smoother than the desired solution. Even if $(1.3)$ has a solution $x_{0}(t)$ in $C[0, T]$, it may not approximate $x(t ; \varepsilon)$ uniformly for all $0 \leq t \leq T$ as $\varepsilon \rightarrow 0$, especially if $x_{0}(0) \neq \lim _{\varepsilon \rightarrow 0} x(t ; \varepsilon)$. In this situation, a second term in (1.2) is needed to correct this nonuniformity. Thus one introduces a new scaled variable with a different magnitude in order to obtain a uniformly valid approximation. The idea is that if the initial layer region is described in terms of the new time scale, no rapid variation in the solution should be exhibited. The additive decomposition singular perturbation method will be employed to analyze the asymptotic solution of (1.1).

Solutions of problems from many branches of applied sciences have been successfully approximated using singular perturbation techniques. When the kernel $a(t, s)$ is independent of $t$ and $s$, problem (1.1) reduces to a singularly perturbed fractional integral. Emerging and increasing use of fractional integrals in physical processes brings about the need to have available and appropriate corresponding singular perturbation techniques. The following operators ${ }_{s} J_{t}^{\gamma}$ and ${ }_{s} D_{t}^{\gamma}$ define the fractional integral and the fractional derivative in the sense of Riemann-Liouville, respectively, of order $\gamma, \varsigma<\gamma<1$ :

$$
\begin{aligned}
{ }_{\varsigma} J_{t}^{\gamma} \phi(t) & :=\frac{1}{\Gamma(\gamma)} \int_{\varsigma}^{t}(t-s)^{\gamma-1} \phi(s) d s, \quad t \geq \varsigma, \\
{ }_{\varsigma} D_{t}^{\gamma} \phi(t) & :=\frac{1}{\Gamma(1-\gamma)} \frac{d}{d t} \int_{\varsigma}^{t}(t-s)^{-\gamma} \phi(s) d s, \quad t>\varsigma .
\end{aligned}
$$

Singularly perturbed Volterra equations with weakly singular kernels occur in various physical applications including heat transfer and viscoelasticity. In particular, fractional integrals arise in the modelling of physical systems, see [16] and the references therein for more on the applications of fractional integrals.

The following is an example showing how singular perturbation problems arise: consider the problem of determining the temperature of a nonlinearly radiating semi-infinite solid. This is governed by the initial-boundary value problem

$$
\begin{gathered}
T_{t}(x, t)=T_{x x}(x, t), \quad x>0, t>0, \\
T_{x}(0, t)=\gamma T^{n}(0, t)-\psi(t), \quad t>0, n \geq 1, \\
T(x, 0)=0, \quad x \geq 0, \\
\lim _{x \rightarrow \infty} T(x, t)=0, \quad t \geq 0 .
\end{gathered}
$$

The function $T(x, t)$ is the temperature. It is assumed that at the point $x=0$, heat is radiated away at a rate proportional to the $n$th power of the temperature. The constant $\gamma$ is the radiation constant; it represents the ratio of the radiative properties to the conductive properties of the solid material. It is also assumed that an external source generates heat 
at a rate proportional to a given positive function $\psi(t)$. The case $n=1$ corresponds to Newton's law of cooling, while the case $n=4$ corresponds to the Stefan-Boltzmann radiation law. It is known that to determine $T(x, t)$ one needs to determine $T(0, t)$ which satisfies the Volterra integral equation

$$
T(0, t)=\int_{0}^{t} \frac{1}{\sqrt{\pi(t-s)}}\left\{\psi(s)-\gamma T^{n}(0, s)\right\} d s .
$$

If one puts $\gamma T^{n}(0, t)=x^{n}(t)$ in the above equation, one obtains

$$
x(t)=\gamma^{1 / n} \int_{0}^{t} \frac{1}{\sqrt{\pi(t-s)}}\left\{\psi(s)-x^{n}(s)\right\} d s .
$$

Then consider (1.7) for the solid material with a higher thermal loss, that is,

$$
\gamma^{-1 / n} \ll 1
$$

Replacing $\gamma^{-1 / n}$ in (1.7) by $\varepsilon$, the resulting equation is one of the problems considered here.

There is an extensive literature on singularly perturbed differential equations. In the study of singularly perturbed integral equations, integral operators play a subordinate role to differential operators. However, there are difficulties in principle in the construction of asymptotic expansions for integral equations depending on a small parameter. These difficulties are basically due to the nature of the integral operators. Singularly perturbed Volterra integral and integrodifferential equations with continuous kernels have received sufficient attention from researchers compared to singularly perturbed integral and integrodifferential equations with weakly singular kernels, see [13] for a comprehensive survey of literature. Singularly perturbed integral and integrodifferential equations with continuous kernels have inner layer solutions which decay exponentially; this simplifies the analysis. Inner layer solutions corresponding to integral and integrodifferential equations with weakly singular kernels decay algebraically, and therefore the analysis poses some complications. Thus, the rigorous theory of singularly perturbed Volterra integral and integrodifferential equations with weakly singular kernels is still under active investigation. This paper aims at presenting a general method for constructing and disclosing the structure of the formal asymptotic solution of (1.1), and providing the proof for its asymptotic correctness.

The additive decomposition method was first applied to study (1.1) in [1]. A variety of interesting examples including (1.7) has been solved in [1], but the analysis fails to reveal the general structure of the formal approximation and the validity of the given formal approximate solution is not demonstrated. To date, apart from the present paper, there have been no efforts to prove the validity of the formal approximation to nonlinear singularly perturbed Volterra integral equations with weakly singular kernels. The linear version of (1.1) has been studied in [3], where it is shown that the formal asymptotic solution is the sum of a slowly varying function and a rapidly varying function, described in terms of the Mittag-Leffler function. The Mittag-Leffler function (a special function) is an example of functions which decay algebraically at infinity, as pointed in the previous 
paragraph. See [10] for more on the Mittag-Leffler function and its properties. Singularly perturbed linear and nonlinear Volterra integrodifferential equations with weakly singular kernels have been investigated in $[4,5]$, respectively. It is demonstrated in $[4,5]$ that singularly perturbed Volterra integrodifferential equations have wider initial layer width and that formal approximate solutions are asymptotic up to the order equivalent to the order of magnitude of the layer width. The main result here is the proof of asymptotic correctness.

In the application of the additive decomposition method to Volterra equations with weakly singular kernels, there are abnormalities arising when balancing terms of similar orders of $\varepsilon$. This occurs when $\alpha$ is not specified and one tries to derive the first (and higher) order terms in the formal solution. But it is known that in the description of some physical processes it is possible to neglect the influence of a number of parameters and not lose valuable information regarding the basic regularities of the process in question. Hence, this paper will restrict attention to the leading order solution.

In the next section, assumptions imposed on the data, and some known results which are applied later in the analysis are presented. In Section 3, the application of the additive decomposition technique to integral equations of type (1.1) is described and also the leading order formal solution derived under the stated conditions. Section 4 contains the proof that the contents of the formal solution have the required properties and those properties imposed during the derivation. It is also shown in Section 4 that if $x_{0}(t ; \varepsilon)$ satisfies (1.1) approximately with a residual $\rho(t ; \varepsilon)$, then $\rho(t ; \varepsilon)=O(\varepsilon), \varepsilon \rightarrow 0$. Finally, in Section 5, we present the theorem on asymptotic correctness which states that under given conditions, if $x_{0}(t ; \varepsilon)$ is a formal approximate solution of $(1.1)$ and $x(t ; \varepsilon)$ is the exact solution, then $\left|x(t ; \varepsilon)-x_{0}(t ; \varepsilon)\right|=O(\varepsilon), \varepsilon \rightarrow 0$. An example is presented in Section 6 to demonstrate the use of the results obtained here.

\section{Hypothesis and mathematical preliminaries}

In the analysis presented, it is assumed that

$\left(\mathrm{H}_{g}\right)$ the function $g(t) \in C^{2}[0, T]$ and is such that $g(0)=0$;

$\left(\mathrm{H}_{a}\right) a(t, s)$ is a $C^{1}$ function on $0 \leq s \leq t \leq T$ with $a(t, t)=-1$;

$\left(\mathrm{H}_{f}\right)$ the function $\psi \mapsto f(\psi, t)$ is at least twice continuously differentiable and that $\partial_{1} f(\psi, t) \neq 0$ for all $\psi$.

The following results will be applied in the presentation.

The first result is that of applying the Mellin transformation to the asymptotic evaluation of integrals. One will find a detailed discussion in $[6,15]$. The Parseval formula for Mellin transforms which will be applied in Section 4 is particularly presented.

The Mellin transform of a locally integrable function $y(t)$ on $(0, \infty)$ is defined by

$$
M[y ; z]:=x(z)=\int_{0}^{\infty} t^{z-1} y(t) d t
$$

when the integral converges. The functions $y(t)$ and $x(z)$ are called the Mellin transform pair. 
Theorem 2.1. Suppose that $M[f ; 1-z]$ and $M[h ; z]$ are defined and holomorphic, each in some vertical strip, whose boundary is determined by the analytical structure of the corresponding function as $t \rightarrow 0$ and as $t \rightarrow \infty$. Suppose further that these strips overlap. Then the integral

$$
I(t)=\int_{0}^{\infty} f(z) h(t z) d z
$$

can be described in terms of the Mellin-Barnes integral, known as the Parseval formula

$$
I(t)=\frac{1}{2 \pi i} \int_{c-i \infty}^{c+i \infty} t^{-z} M[f ; 1-z] M[h ; z] d z,
$$

where $\operatorname{Re}(z)=c$ lies in the overlapping strip.

The second result is that of Abel inversion formula which follows from the results in [11]. Let $0<\delta<1$ and define the operator

$$
\left(D^{\delta} y\right)(t):=\frac{1}{\Gamma(1-\delta)} \frac{d}{d t} \int_{0}^{t} \frac{1}{(t-s)^{\delta}} y(s) d s .
$$

Theorem 2.2. If $y(t)$ is continuous on $[0, T]$ and differentiable on $(0, T]$ with $y(0)=0$, then $D^{\delta}(y)(t)$ is also continuous on $[0, T]$ and is given by

$$
\left(D^{\delta} y\right)(t)=\frac{1}{\Gamma(1-\delta)} \int_{0}^{t} \frac{1}{(t-s)^{\delta}} y^{\prime}(s) d s
$$

The third result is the property of Riemann-Liouville which says that the RiemannLiouville fractional differentiation operator is a left inverse to the Riemann-Liouville fractional integration operator of the same order.

Theorem 2.3. For $\delta>0$ and $t>0$,

$$
D^{\delta}\left(J^{\delta} \phi(t)\right)=\phi(t)
$$

The proof can be found in many books on fractional derivatives and fractional integrals.

The fourth and last result is a nonlinear generalization of Gronwall's inequality and has been proved in [8].

Theorem 2.4. Let $\phi, \psi:[\iota, \zeta) \rightarrow[0, \infty), \varphi:[\iota, \zeta) \times[0, \infty) \rightarrow[0, \infty)$ be continuous such that

$$
0 \leq \varphi(t, u)-\varphi(t, v) \leq M(t, u)(u-v), \quad t \in[\iota, \zeta), 0 \leq v \leq u,
$$

where $M$ is nonnegative and continuous on $[\iota, \zeta) \times[0, \infty)$. Then, for every nonnegative continuous solution of the inequality

$$
y(t) \leq \phi(t)+\psi(t) \int_{\iota}^{t} \varphi(s, y(s)) d s, \quad t \in[\iota, \zeta),
$$


the following estimate holds:

$$
y(t) \leq \phi(t)+\psi(t) \int_{\iota}^{t} \varphi(s, \phi(s)) \exp \left(\int_{s}^{t} M(\sigma, \phi(\sigma)) \psi(\sigma) d \sigma\right) d s
$$

for all $t$ in $[\iota, \zeta)$.

\section{Heuristic analysis and formal solution}

To start, one seeks an asymptotic solution $x(t ; \varepsilon)$ in the form

$$
x(t ; \varepsilon)=u(t ; \varepsilon)+v\left(\frac{t}{\varepsilon^{\eta}} ; \varepsilon\right), \quad \eta>0,
$$

and requires that

$$
\lim _{\tau \rightarrow \infty} v(\tau ; \varepsilon)=0, \quad \tau=\frac{t}{\varepsilon^{\eta}}
$$

The inner layer function $v\left(t / \varepsilon^{\eta} ; \varepsilon\right)$ corrects the nonuniformity in the initial layer. It is assumed that $u(t ; \varepsilon)$ and $v(\tau ; \varepsilon)$ have asymptotic expansions of the form

$$
u(t ; \varepsilon) \sim \sum_{n=0}^{\infty} \varepsilon^{n} u_{n}(t), \quad v(\tau ; \varepsilon) \sim \sum_{n=0}^{\infty} \varepsilon^{n \eta} v_{n}(\tau)
$$

as $\varepsilon \rightarrow 0$, so that

$$
x(t ; \varepsilon) \sim \sum_{n=0}^{\infty} \varepsilon^{n} u_{n}(t)+\sum_{n=0}^{\infty} \varepsilon^{n \eta} v_{n}\left(\frac{t}{\varepsilon^{\eta}}\right) .
$$

Moreover, for all $n \geq 0$,

$$
\lim _{\tau \rightarrow \infty} v_{n}(\tau)=0
$$

The substitution of (3.4) into (1.1), the expression of all terms in terms of $\tau$, and the examination of the dominant balance in the relation yield

$$
\operatorname{Ord}\left(\varepsilon^{\eta+\eta(\alpha-1)}\right)=\operatorname{Ord}(\varepsilon), \quad \varepsilon \longrightarrow 0
$$

Hence one chooses

$$
\eta=\frac{1}{\alpha}
$$

Thus, the singularly perturbed equation (1.1) possesses an initial layer width of order $\left(\varepsilon^{1 / \alpha}\right)$. This means that the solution $x(t ; \varepsilon)$ of $(1.1)$ is slowly varying for $O\left(\varepsilon^{1 / \alpha}\right) \leq t \leq T$, as $\varepsilon \rightarrow 0$, but changes rapidly on a small interval $0 \leq t \leq O\left(\varepsilon^{1 / \alpha}\right)$. This small interval of rapid 
change is what is referred to as the inner layer, initial layer, or layer of rapid transition and the region of slow variation of $x(t ; \varepsilon)$ is what is referred to as the outer region. The choice $\eta=1 / \alpha$ implies that (1.1) has a narrower initial layer compared to a similar equation with a continuous kernel and integrodifferential equations with weakly singular kernels.

3.1. Derivation of the formal approximate solution. The results in this section are summarized in the following proposition.

Proposition 3.1. Suppose that $\left(H_{g}\right),\left(H_{a}\right)$, and $\left(H_{f}\right)$ hold. Suppose also that

$$
v_{0}(\tau) \sim c_{0} \tau^{-\beta_{0}}, \quad \tau \longrightarrow \infty,
$$

for some constants $c_{0}$ and $\beta_{0}$ such that $0<\beta_{0}<1$. Assume

$$
x_{0}(t ; \varepsilon)=u_{0}(t)+v_{0}\left(\frac{t}{\varepsilon^{\eta}}\right), \quad \eta=\frac{1}{\alpha},
$$

satisfies (1.1) approximately, with a residual $\rho(t ; \varepsilon)$ such that

$$
\rho(t ; \varepsilon)=o(1), \quad \varepsilon \longrightarrow 0,0 \leq t \leq T .
$$

Then (1.1) has a formal approximate solution given by (3.9), where $u_{0}$ and $v_{0}$ satisfy corresponding nonlinear Volterra equations with weakly singular kernels.

Proof. Suppose that $x_{0}(t ; \varepsilon)$ satisfies $(1.1)$ approximately with a residual $\rho(t ; \varepsilon)$; then

$$
\rho(t ; \varepsilon)+\varepsilon u_{0}(t)+\varepsilon v_{0}\left(\frac{t}{\varepsilon^{\eta}}\right)=g(t)+\frac{1}{\Gamma(\alpha)} \int_{0}^{t} \frac{a(t, s)}{(t-s)^{1-\alpha}} f\left(u_{0}(s)+v_{0}\left(\frac{s}{\varepsilon^{\eta}}\right), s\right) d s
$$

equivalently,

$$
\begin{aligned}
\rho(t ; \varepsilon)= & -\varepsilon u_{0}(t)-\varepsilon v_{0}\left(\frac{t}{\varepsilon^{\eta}}\right)+g(t) \\
& +\frac{1}{\Gamma(\alpha)} \int_{0}^{t} \frac{a(t, s)}{(t-s)^{1-\alpha}} f\left(u_{0}(s), s\right) d s \\
& +\frac{1}{\Gamma(\alpha)} \int_{0}^{t} \frac{a(t, s)}{(t-s)^{1-\alpha}}\left\{f\left(u_{0}(s)+v_{0}\left(\frac{s}{\varepsilon^{\eta}}\right), s\right)-f\left(u_{0}(s), s\right)\right\} d s .
\end{aligned}
$$

The last integral in (3.12) can be written as

$$
I_{0}(t)=\frac{t^{\alpha}}{\Gamma(\alpha)} \int_{0}^{1} \frac{a(t, t \theta)}{(1-\theta)^{1-\alpha}}\left\{f\left(u_{0}(t \theta)+v_{0}\left(\frac{t \theta}{\varepsilon^{\eta}}\right), t \theta\right)-f\left(u_{0}(t \theta), t \theta\right)\right\} d \theta .
$$

Using Taylor's theorem, one writes $I_{0}$ as

$$
I_{0}(t)=\frac{t^{\alpha}}{\Gamma(\alpha)} \int_{0}^{1} \frac{a(t, t \theta)}{(1-\theta)^{1-\alpha}}\left\{f_{1}\left(u_{0}(t \theta), t \theta\right) v_{0}\left(\frac{t \theta}{\varepsilon^{\eta}}\right)+v_{0}^{2}\left(\frac{t \theta}{\varepsilon^{\eta}}\right) \phi(t, \theta ; \varepsilon)\right\} d \theta,
$$


where

$$
\phi(t, \theta ; \varepsilon)=\int_{0}^{1}(1-\mu) f_{11}\left(u_{0}(t \theta)+\mu v_{0}\left(\frac{t \theta}{\varepsilon^{\eta}}\right), t \theta\right) d \mu .
$$

Thus, applying (3.8) and the dominated convergence theorem, (3.12) is equivalent to

$$
\begin{aligned}
\rho(t ; \varepsilon)= & -\varepsilon u_{0}(t)-\varepsilon v_{0}\left(\frac{t}{\varepsilon^{\eta}}\right)+g(t) \\
& +\frac{1}{\Gamma(\alpha)} \int_{0}^{t} \frac{a(t, s)}{(t-s)^{1-\alpha}} f\left(u_{0}(s), s\right) d s \\
& +\frac{c_{0} \varepsilon^{\eta \beta_{0}}}{\Gamma(\alpha)} t^{\alpha-\beta_{0}} \int_{0}^{1} \frac{a(t, t \theta)}{(1-\theta)^{1-\alpha} \theta^{\beta_{0}}} f_{1}\left(u_{0}(t \theta), t \theta\right) d \theta+o(1),
\end{aligned}
$$

as $\varepsilon \rightarrow 0$. The leading order outer equation is then obtained by fixing $t>0$ in the above equation and letting $\varepsilon$ tend to zero. If $\rho(t ; \varepsilon)=o(1)$ as $\varepsilon \rightarrow 0$, one sees that

$$
0=g(t)+\frac{1}{\Gamma(\alpha)} \int_{0}^{t} \frac{a(t, s)}{(t-s)^{1-\alpha}} f\left(u_{0}(s), s\right) d s
$$

The equation governing the leading order inner layer solution is obtained by substituting (3.17) into (3.12), expressing all terms in terms of $\tau$, dividing by the dominant power of $\varepsilon$, fixing $\tau$, and letting $\varepsilon$ tend to zero. If $\rho\left(\varepsilon^{\eta} \tau ; \varepsilon\right)=o(1)$ as $\varepsilon \rightarrow 0$ for fixed $\tau>0$, then

$$
v_{0}(\tau)+u_{0}(0)=\frac{a(0,0)}{\Gamma(\alpha)} \int_{0}^{\tau}(\tau-\sigma)^{\alpha-1}\left\{f\left(u_{0}(0)+v_{0}(\sigma), 0\right)-f\left(u_{0}(0), 0\right)\right\} d \sigma, \quad \tau \geq 0 .
$$

If $u_{0}(t)$ satisfies (3.17) and $v_{0}(\tau)$ obeys (3.18), it follows from (3.12) that

$$
\begin{aligned}
\rho(t ; \varepsilon)= & \varepsilon u_{0}(0)-\varepsilon u_{0}(t) \\
& -\frac{1}{\Gamma(\alpha)} \int_{0}^{t} \frac{a(0,0)}{(t-s)^{1-\alpha}}\left\{f\left(u_{0}(0)+v_{0}\left(\frac{s}{\varepsilon^{\eta}}\right), 0\right)-f\left(u_{0}(0), 0\right)\right\} d s \\
& +\frac{1}{\Gamma(\alpha)} \int_{0}^{t} \frac{a(t, s)}{(t-s)^{1-\alpha}}\left\{f\left(u_{0}(s)+v_{0}\left(\frac{s}{\varepsilon^{\eta}}\right), s\right)-f\left(u_{0}(s), s\right)\right\} d s
\end{aligned}
$$

Remark 3.2. Following the above analysis, the derivation of the equations governing higher-order terms in (3.4) depends on the actual value of $\eta$. Thus one will be interested in modifying the present methodology to include higher-order terms as well.

\section{Properties of the formal solution}

It is shown in this section that there are unique solutions $u_{0}(t)$ and $v_{0}(\tau)$ of (3.17) and (3.18), and that they have the important properties assumed in their derivation.

Equation (3.17) is a Volterra integral equation of the first kind for $u_{0}(t)$. If one puts

$$
f\left(u_{0}(t), t\right)=\varphi(t), \quad 0 \leq t \leq T,
$$


the resulting equation

$$
0=g(t)+\frac{1}{\Gamma(\alpha)} \int_{0}^{t} \frac{a(t, s)}{(t-s)^{1-\alpha}} \varphi(s) d s, \quad t \geq 0
$$

is a linear Volterra equation of the first kind. A unique solution $\varphi(t) \in C^{1}[0, T]$ exists under given conditions $\left(\mathrm{H}_{g}\right)$ and $\left(\mathrm{H}_{a}\right)$, see [11]. Then the existence and uniqueness of a continuous solution $u_{0}(t)$ of (4.1) follow from the implicit function theorem and the fact that $\partial_{1} f\left(u_{0}(t), t\right) \neq 0$. The cases $a(t, s)=k$, a constant, and $a(t, s)=a(t-s)$ would give the function $\varphi(t)$ exactly. Numerical approximation of $u_{0}(t)$ from (4.1) should then be easy.

Proposition 4.1. Suppose that $\left(H_{g}\right),\left(H_{a}\right)$, and $\left(H_{f}\right)$ hold. Then (3.18) has a $C^{\infty}$ solution $v_{0}$ satisfying

$$
v_{0}(\tau) \sim \frac{-u_{0}(0)}{\Gamma(1-\alpha)} \tau^{-\alpha}, \quad \tau \longrightarrow \infty
$$

Proof. Equation (3.18) cannot be solved explicitly; however, this equation is simpler than the original equation in the sense that the forcing function and the kernel have simpler forms. Existence and uniqueness of solutions of (3.18) for $\tau \geq 0$ are well known, see, for example, $[7,11,12,14]$.

To prove the asymptotic behavior (4.3) of $v_{0}(\tau)$, as $\tau \rightarrow \infty$, the presentation below follows the analysis in [6, Chapter 4]. Consider (3.18) and let

$$
I(\tau)=\frac{-1}{\Gamma(\alpha)} \int_{0}^{\tau}(\tau-\sigma)^{\alpha-1}\left\{f\left(u_{0}(0)+v_{0}(\sigma), 0\right)-f\left(u_{0}(0), 0\right)\right\} d \sigma,
$$

which can equivalently be written as

$$
I(\tau)=-\tau^{\alpha} I_{\alpha}(\tau)
$$

Here,

$$
I_{\alpha}(\tau)=\int_{0}^{\infty} \phi(\theta) \psi(\tau \theta) d \theta
$$

where

$$
\begin{gathered}
\phi(\theta)=\left\{\begin{array}{lc}
\frac{1}{\Gamma(\alpha)}(1-\theta)^{\alpha-1} & 0 \leq \theta<1, \\
0 & 1 \leq \theta,
\end{array}\right. \\
\psi(\theta \tau)=f\left(u_{0}(0)+v_{0}(\theta \tau), 0\right)-f\left(u_{0}(0), 0\right) .
\end{gathered}
$$

Thus, to determine the asymptotic behavior of $v_{0}(\tau), \tau \rightarrow \infty$, one needs to determine the asymptotic behavior of $I_{\alpha}(\tau), \tau \rightarrow \infty$, and then multiply by $-\tau^{\alpha}$. The form of the integral in (4.6) suggests the application of Mellin transform in the determination of an asymptotic expansion of $I_{\alpha}(\tau), \tau \rightarrow \infty$. This is a well known and powerful technique. Since 
this technique involves asymptotic behavior of functions in the integrand, one should note that Taylor's theorem implies that $v_{0}(\tau)$ and $\psi(\tau)$ are $O$-equivalent:

$$
\psi(\tau)=\operatorname{Ord}\left(v_{0}(\tau)\right), \quad \tau \longrightarrow 0, \tau \longrightarrow \infty .
$$

We know from (3.18) that $v_{0}(\tau)=O(1), \tau \rightarrow 0$, and we have assumed in (3.8) that $v_{0}(\tau)=$ $O\left(\tau^{-\beta_{0}}\right), \tau \rightarrow \infty$.

Now, suppose that $M[\phi ; 1-z]$ and $M[\psi ; z]$ are defined and holomorphic, each in some vertical strip determined by the asymptotic behaviors of $\phi$ and $\psi$. Since $I_{\alpha}(\tau)$ is absolutely convergent, these vertical strips overlap. Let $\operatorname{Re}\{z\}=c$ lay in the overlapping strip; then the Parseval formula (2.3) implies that

$$
I_{\alpha}(\tau)=\frac{1}{2 \pi i} \int_{c-i \infty}^{c+i \infty} \tau^{-z} M[\phi ; 1-z] M[\psi ; z] d z
$$

One observes from (2.1) that

$$
M[\phi ; 1-z]=\frac{\Gamma(1-z)}{\Gamma(1+\alpha-z)},
$$

and therefore

$$
I_{\alpha}(\tau)=\frac{1}{2 \pi i} \int_{c-i \infty}^{c+i \infty} \tau^{-z} M[\psi ; z] \frac{\Gamma(1-z)}{\Gamma(1+\alpha-z)} d z .
$$

The asymptotic evaluation of (4.11) as $\tau \rightarrow \infty$ involves the asymptotic behavior of $\psi(\tau), \tau \rightarrow \infty$, which in turn involves the asymptotic behavior of $v_{0}(\tau)$, the result set out to be obtained. Thus the approach will be to employ the assumed leading order asymptotic behavior of $v_{0}(\tau)$ in (3.8). Then the asymptotic expansion of $I_{\alpha}$ can be derived using Mellin transform procedure. This will involve some unknown parameters which will be determined by requiring the derived expansion to coincide with the left-hand side of (3.18) which involves the assumed expansion of $v_{0}$.

The asymptotic relation in (4.8) implies that

$$
\psi(\tau) \sim c_{0} \tau^{-\beta_{0}}, \quad \tau \longrightarrow \infty, \text { for some positive } \beta_{0}<1 .
$$

It can be shown that $M[\psi ; z]$ has a simple pole at $z=\beta_{0}$ and the singular part of the Laurent expansion of $M[\psi ; z]$ about this point is given by

$$
\frac{-c_{0}}{z-\beta_{0}} \text {. }
$$

To compute the asymptotic behavior of $I_{\alpha}$, the vertical path is displaced to the right. In doing so, the pole implied by $M[\psi ; z]$ is encountered before that of $\Gamma(1-z)$ at $z=1$ since $\beta_{0}<1$, and hence it provides the leading order contribution. Computing the relevant residues, one finds that

$$
v_{0}(\tau)+u_{0}(0) \sim-c_{0} M\left[\psi ; 1-\beta_{0}\right] \tau^{\alpha-\beta_{0}}=-c_{0} \frac{\Gamma\left(1-\beta_{0}\right)}{\Gamma\left(1+\alpha-\beta_{0}\right)} \tau^{\alpha-\beta_{0}} .
$$


The dominant term balancing argument then implies

$$
\alpha=\beta_{0}, \quad c_{0}=\frac{-u_{0}(0)}{\Gamma(1-\alpha)} .
$$

Therefore

$$
v_{0}(\tau) \sim \frac{-u_{0}(0)}{\Gamma(1-\alpha)} \tau^{-\alpha}, \quad \tau \longrightarrow \infty
$$

This result agrees with that obtained in [3] for $f(x, s)=x$ using the explicit solution in terms of the Mittag-Leffler function.

Therefore, up to the leading order, the formal approximate solution $x_{0}(t ; \varepsilon)$ of $(1.1)$ is given by (3.9), where $u_{0}(t)$ and $v_{0}(\tau)$ are solutions of (3.17) and (3.18), respectively. Having established the existence and uniqueness of the approximate solution, the following proposition bounds the residual $\rho(t ; \varepsilon)$.

Proposition 4.2. Suppose $\rho(t ; \varepsilon)$ satisfies (3.19); then there exist positive constants $c_{1}$, which does not dependent on $\varepsilon$, and $\varepsilon_{0}$ such that

$$
\left|\left(D^{\alpha} \rho\right)(t ; \varepsilon)\right| \leq c_{1} \varepsilon, \quad \varepsilon \longrightarrow 0,
$$

for all $0 \leq t \leq T$ and all $0<\varepsilon \leq \varepsilon_{0}$.

Proof. An argument similar to the one used in (3.14) and (3.15) for the Taylor expansion implies that one can write (3.19) as

$$
\begin{aligned}
\rho(t ; \varepsilon)= & \varepsilon u_{0}(0)-\varepsilon u_{0}(t) \\
& -\frac{t^{\alpha}}{\Gamma(\alpha)} \int_{0}^{1} \frac{a(0,0)}{(1-s)^{1-\alpha}}\left\{f_{1}\left(u_{0}(0), 0\right) v_{0}\left(\frac{t s}{\varepsilon^{\eta}}\right)-\phi(t, s ; \varepsilon) v_{0}^{2}\left(\frac{t s}{\varepsilon^{\eta}}\right)\right\} d s \\
& +\frac{t^{\alpha}}{\Gamma(\alpha)} \int_{0}^{1} \frac{a(t, t s)}{(1-s)^{1-\alpha}}\left\{f_{1}\left(u_{0}(t s), t s\right) v_{0}\left(\frac{t s}{\varepsilon^{\eta}}\right)+\phi(t, s ; \varepsilon) v_{0}^{2}\left(\frac{t s}{\varepsilon^{\eta}}\right)\right\} d s,
\end{aligned}
$$

where the function $\phi$ is as given in (3.15). The dominated convergence theorem and (4.16) imply that when $\varepsilon$ is sufficiently small, $\rho(t ; \varepsilon)$ can be approximated by

$$
\begin{aligned}
\rho(t ; \varepsilon)= & \varepsilon u_{0}(0)-\varepsilon u_{0}(t)-\varepsilon u_{0}(0) f_{1}\left(u_{0}(0), 0\right) \\
& -\frac{\varepsilon u_{0}(0)}{\Gamma(\alpha) \Gamma(1-\alpha)} \int_{0}^{1} \frac{a(t, t s)}{(1-s)^{1-\alpha} s^{\alpha}} f_{1}\left(u_{0}(t s), t s\right) d s .
\end{aligned}
$$

Thus $\rho(t ; \varepsilon)$ is continuous for all $0 \leq t \leq T$ and all $0 \leq \varepsilon$ with $\rho(0 ; \varepsilon)=0$. It is also differentiable for all $t>0$. Thus, Theorem 2.2 implies that there exist positive constants $c_{1}$ and $\varepsilon_{0}$, where $c_{1}$ depends on $T$, such that for all $0 \leq t \leq T$ and all $0<\varepsilon \leq \varepsilon_{0}$,

$$
\left|\left(D^{\alpha} \rho\right)(t ; \varepsilon)\right| \leq c_{1} \varepsilon
$$




\section{Proof of asymptotic correctness}

In this section, it is established that the formal approximation defined in (3.9) is indeed an asymptotic approximation to a solution $x(t ; \varepsilon)$ of $(1.1)$. The method is to adapt the theory of [9] on developing a rigorous theory of singular perturbation. The main result in this paper is the following.

Theorem 5.1. Suppose that $\left(H_{g}\right),\left(H_{a}\right)$, and $\left(H_{f}\right)$ hold. Then (1.1) has a continuous solution $x(t ; \varepsilon)$ with the property that if $x_{0}(t ; \varepsilon)$ is the formal asymptotic solution, then there are constants $c^{\star}$, which does not depend on $\varepsilon$, and $\varepsilon_{0}$ such that

$$
\left|x(t ; \varepsilon)-x_{0}(t ; \varepsilon)\right| \leq c^{\star} \varepsilon
$$

for all $0 \leq t \leq T$ and $0<\varepsilon \leq \varepsilon_{0}$.

Proof. Define the remainder

$$
\chi(t ; \varepsilon)=x(t ; \varepsilon)-x_{0}(t ; \varepsilon), \quad 0 \leq t \leq T .
$$

Substituting this into (1.1) and using (3.4) gives

$$
\varepsilon \chi(t ; \varepsilon)=\rho(t ; \varepsilon)+\frac{1}{\Gamma(\alpha)} \int_{0}^{t} \frac{a(t, s)}{(t-s)^{1-\alpha}}\left\{f\left(x_{0}(s ; \varepsilon)+\chi(s ; \varepsilon), s\right)-f\left(x_{0}(s ; \varepsilon), s\right)\right\} d s .
$$

Applying Taylor's theorem, one has an equivalent equation

$$
\begin{aligned}
\varepsilon \chi(t ; \varepsilon)= & \rho(t ; \varepsilon)+\frac{1}{\Gamma(\alpha)} \int_{0}^{t} \frac{a(t, s)}{(t-s)^{1-\alpha}} \partial_{1} f\left(x_{0}, s ; \varepsilon\right) \chi(s ; \varepsilon) d s \\
& +\frac{1}{\Gamma(\alpha)} \int_{0}^{t} \frac{a(t, s)}{(t-s)^{1-\alpha}} \varphi\left(\chi, x_{0}, s ; \varepsilon\right) d s,
\end{aligned}
$$

where

$$
\varphi\left(\chi, x_{0}, t ; \varepsilon\right)=\chi(t ; \varepsilon)^{2} \int_{0}^{1}(1-\mu) \partial_{11} f\left(x_{0}+\mu \chi, t ; \varepsilon\right) d \mu .
$$

One can also write (5.4) as

$$
\begin{aligned}
\varepsilon \chi(t ; \varepsilon)= & \rho(t ; \varepsilon)+\frac{1}{\Gamma(\alpha)} \int_{0}^{t} \frac{a(0,0)}{(t-s)^{1-\alpha}} \chi(s ; \varepsilon) d s+\frac{1}{\Gamma(\alpha)} \int_{0}^{t} \frac{k(t, s ; \varepsilon)}{(t-s)^{1-\alpha}} \chi(s ; \varepsilon) d s \\
& +\frac{1}{\Gamma(\alpha)} \int_{0}^{t} \frac{a(t, s)}{(t-s)^{1-\alpha}} \varphi\left(\chi, x_{0}, s ; \varepsilon\right) d s,
\end{aligned}
$$

where

$$
k(t, s ; \varepsilon)=a(t, s) \partial_{1} f\left(x_{0}, s ; \varepsilon\right)-a(0,0) .
$$

Note that $\partial_{1} f$ and $k$ are both regular with respect to $\varepsilon$ as $\varepsilon \rightarrow 0$. Applying the operator $D^{\alpha}$ (defined in (2.4)) to both sides of (5.6) gives

$$
\chi(t ; \varepsilon)=G(\chi)(t ; \varepsilon)+\int_{0}^{t} h(t, s ; \varepsilon) \chi(s ; \varepsilon) d s, \quad 0 \leq t \leq T,
$$


where $\chi \mapsto G(\chi)$ is given by

$$
G(\chi)(t ; \varepsilon)=\left(D^{\alpha} \rho\right)(t ; \varepsilon)-\varepsilon\left(D^{\alpha} \chi\right)(t ; \varepsilon)+\int_{0}^{t} \tilde{h}(t, s) \varphi\left(\chi, x_{0}, s ; \varepsilon\right) d s,
$$

with

$$
\begin{aligned}
h(t, s ; \varepsilon) & =\frac{1}{\Gamma(\alpha) \Gamma(1-\alpha)} \frac{\partial}{\partial t} \int_{s}^{t} \frac{k(\sigma, s ; \varepsilon)}{(t-\sigma)^{\alpha}(\sigma-s)^{1-\alpha}} d \sigma \\
\tilde{h}(t, s) & =\frac{1}{\Gamma(\alpha) \Gamma(1-\alpha)} \frac{\partial}{\partial t} \int_{s}^{t} \frac{a(\sigma, s)}{(t-\sigma)^{\alpha}(\sigma-s)^{1-\alpha}} d \sigma
\end{aligned}
$$

obtained using Theorem 2.3 and Fubini's theorem. It can be shown that both $h(t, s ; \varepsilon)$ and $\tilde{h}(t, s)$ are continuous functions on $0 \leq s \leq t \leq T$.

Let $H(t, s ; \varepsilon)$ be the resolvent kernel for $h(t, s ; \varepsilon)$; then $H(t, s ; \varepsilon)$ is given by

$$
H(t, s ; \varepsilon)=h(t, s ; \varepsilon)+\int_{s}^{t} h(t, \sigma ; \varepsilon) H(\sigma, s ; \varepsilon) d \sigma,
$$

and (5.8) can equivalently be given by the variation of the constants formula

$$
\chi(t ; \varepsilon)=G(\chi)(t ; \varepsilon)+\int_{0}^{t} H(t, s ; \varepsilon) G(\chi)(s ; \varepsilon) d s, \quad 0 \leq t \leq T
$$

The above equation is equivalent to

$$
\begin{aligned}
\chi(t ; \varepsilon)= & \left(D^{\alpha} \rho\right)(t ; \varepsilon)-\varepsilon\left(D^{\alpha} \chi\right)(t ; \varepsilon) \\
& +\int_{0}^{t} H(t, s ; \varepsilon)\left\{\left(D^{\alpha} \rho\right)(s ; \varepsilon)-\varepsilon\left(D^{\alpha} \chi\right)(s ; \varepsilon)\right\} d s \\
& +\int_{0}^{t} \varphi\left(\chi, x_{0}, s ; \varepsilon\right) \tilde{H}(t, s ; \varepsilon) d s,
\end{aligned}
$$

where

$$
\tilde{H}(t, s ; \varepsilon)=\tilde{h}(t, s)+\int_{s}^{t} H(t, \sigma ; \varepsilon) \tilde{h}(\sigma, s) d \sigma
$$

is continuous on $0 \leq s \leq t \leq T$.

It is known that $\chi(t ; \varepsilon)$ is continuous with $\chi(0 ; \varepsilon)=0, \varepsilon>0$. Moreover, (4.20) and (5.8) imply that, when $\varepsilon=0$,

$$
\chi(t ; 0)=\int_{0}^{t} h(t, s ; 0) \chi(s ; 0) d s+\int_{0}^{t} \tilde{h}(t, s) \varphi\left(\chi, x_{0}, s ; 0\right) d s .
$$

Thus, Theorem 2.2 implies that there exist positive constants $c_{2}$ (depending on $T$ ) and $\varepsilon_{0}$ such that

$$
\left|\left(D^{\alpha} \chi\right)(t ; \varepsilon)\right| \leq c_{2}, \quad 0 \leq t \leq T, 0<\varepsilon \leq \varepsilon_{0} .
$$


It then follows from (5.13), Proposition 4.2, and (5.5) that for all $t$ in $[0, T]$ and all $\varepsilon$ in $\left(0, \varepsilon_{0}\right]$,

$$
|\chi(t ; \varepsilon)| \leq\left(c_{1} \varepsilon+c_{2} \varepsilon\right)(1+\|H\| t)+\|\tilde{H}\| \int_{0}^{t} c_{3}|\chi(s ; \varepsilon)|^{2} d s
$$

where

$$
\begin{aligned}
& \|H\|=\max _{0 \leq s \leq t \leq T, \varepsilon \geq 0}|H(t, s ; \varepsilon)|, \\
& \|\tilde{H}\|=\max _{0 \leq s \leq t \leq T, \varepsilon \geq 0}|\tilde{H}(t, s ; \varepsilon)|, \\
& c_{3}=\max _{\substack{0 \leq t \leq T \\
0<\mu<1, \varepsilon \geq 0}}\left|\partial_{11} f\left(x_{0}+\chi \mu, t ; \varepsilon\right)\right| .
\end{aligned}
$$

Application of Theorem 2.4 yields the required result.

Remark 5.2. The analysis in Section 3.1 for higher-order terms depends on the actual value of $\alpha$. But the proof of asymptotic correctness presented in Section 5 holds for all terms in (3.4) and for any value of $\eta$.

\section{Example}

The following example obtained from [16, Chapter 8] is an initial-boundary value problem describing the process of cooling of a semi-infinite body (with higher thermal loss) by radiation:

$$
\begin{gathered}
\frac{\partial}{\partial t} T(x, t)=\frac{\partial^{2}}{\partial x^{2}} T(x, t), \quad x>0, t>0, \\
\frac{\partial}{\partial x} T(0, t)=\frac{1}{\varepsilon} T^{4}(0, t), \quad t>0,0<\varepsilon \ll 1, \\
T(\infty, t)=T(x, 0)=a_{0}, \quad x \geq 0, t \geq 0,
\end{gathered}
$$

where $a_{0}$ is a constant. Here, one is interested in finding the temperature $T(0, t)$ for $t>0$. It can be shown that if $T(x, t)$ satisfies (6.1a) and (6.1c), then

$$
a_{0}-T(0, t)=\frac{1}{\Gamma(1 / 2)} \int_{0}^{t}(t-s)^{-1 / 2} \frac{\partial}{\partial x} T(0, s) d s
$$

Using (6.1b), one obtains

$$
\varepsilon\left(a_{0}-T(0, t)\right)=\frac{1}{\Gamma(1 / 2)} \int_{0}^{t}(t-s)^{-1 / 2} T^{4}(0, s) d s .
$$


Let $T(0, t)=\phi(t)$ and consider the nonlinear singularly perturbed fractional integral equation

$$
\varepsilon \phi(t)=\varepsilon a_{0}-\frac{1}{\Gamma(1 / 2)} \int_{0}^{t}(t-s)^{-1 / 2} \phi^{4}(s) d s, \quad t \geq 0 .
$$

Note that $g$ depends on $\varepsilon$ and that $\left(\mathrm{H}_{g}\right),\left(\mathrm{H}_{a}\right)$, and $\left(\mathrm{H}_{f}\right)$ are satisfied.

The limiting equation obtained by letting $\varepsilon \rightarrow 0$ in (6.4) is

$$
0=\frac{1}{\Gamma(1 / 2)} \int_{0}^{t}(t-s)^{-1 / 2} \phi_{0}^{4}(s) d s, \quad t \geq 0
$$

This implies $\phi_{0}(t)=0, t \geq 0$. Thus the reduced equation has a qualitative behavior different from the original problem. In particular, $\phi_{0}(0) \neq \phi(0)$ when $a_{0} \neq 0$. Thus, when $a_{0} \neq 0$, the solution $\phi(t ; \varepsilon)$ of $(6.4)$ is not everywhere close to the solution of the unperturbed equation, obtained by letting $\varepsilon \rightarrow 0$. Therefore (6.4) is singularly perturbed.

To analyze (6.4) consider the approximation given in (3.9) with $\eta=2$. The leading order outer solution $u_{0}(t)$ follows from (3.17) as

$$
u_{0}(t)=0, \quad 0 \leq t \leq T
$$

The governing equation for the leading order inner layer solution is given by

$$
v_{0}(\tau)=a_{0}-\frac{1}{\Gamma(1 / 2)} \int_{0}^{\tau}(\tau-\sigma)^{-1 / 2} v_{0}^{4}(\sigma) d \sigma, \quad \tau \geq 0 .
$$

This equation cannot be solved exactly for $v_{0}(\tau)$ but it is simpler compared to (6.4) in the sense that it does not contain the parameter $\varepsilon$. Initial investigation of this equation implies that there exists a solution $v_{0}(\tau)$ for all $\tau \geq 0$, such that

$$
0 \leq v_{0}(\tau) \leq a_{0}, \quad \forall \tau \geq 0
$$

Further analysis reveals that the Mellin transform technique can be used to describe the asymptotic behavior of the solution $v_{0}(\tau)$ of (6.7) for large and small values of $\tau$. In particular, applying Proposition 4.1 to (6.7) with

$$
\psi(\tau)=v_{0}^{4}(\tau)
$$

strongly nonlinear implies that $\psi(\tau)=o\left(v_{0}(\tau)\right)=O\left(\tau^{-4 \beta_{0}}\right), \tau \rightarrow \infty$. Following the proof of Proposition 4.1 with $\alpha=1 / 2$ reveals that

$$
v_{0}(\tau) \sim \frac{a_{0}}{\sqrt{\pi}} \tau^{-1 / 8}, \quad \tau \longrightarrow \infty .
$$

A similar analysis from [6] implies that

$$
v_{0}(\tau) \sim a_{0}+\frac{2 a_{0}^{4}}{\sqrt{\pi}} \tau^{1 / 2}, \quad \tau \longrightarrow 0 .
$$


These results agree with those obtained in [2]. Thus the uniformly valid asymptotic solution of (6.4) is

$$
\phi(t ; \varepsilon)=v_{0}\left(\frac{t}{\varepsilon^{2}}\right), \quad 0 \leq t \leq T
$$

for all $\varepsilon \geq 0$ and not the $\phi_{0}(t)$.

\section{Acknowledgment}

The author wishes to thank the referees for their helpful and positive comments.

\section{References}

[1] J. S. Angell and W. E. Olmstead, Singularly perturbed Volterra integral equations, SIAM J. Appl. Math. 47 (1987), no. 1, 1-14.

[2] Yu. I. Babenko, Heat and Mass Transfer, Khimiya, Leningrad, 1986.

[3] A. M. Bijura, Singularly perturbed Volterra integral equations with weakly singular kernels, Int. J. Math. Math. Sci. 30 (2002), no. 3, 129-143.

[4] Asymptotics of integrodifferential models with integrable kernels, Int. J. Math. Math. Sci. 2003 (2003), no. 25, 1577-1598.

[5] - Asymptotics of integrodifferential models with integrable kernels. II, Int. J. Math. Math. Sci. 2003 (2003), no. 50, 3153-3169.

[6] N. Bleistein and R. A. Handelsman, Asymptotic Expansions of Integrals, Holt, Rinehart and Winston, New York, 1975.

[7] K. Diethelm and N. J. Ford, Analysis of fractional differential equations, J. Math. Anal. Appl. 265 (2002), no. 2, 229-248.

[8] S. S. Dragomir, On some nonlinear generalizations of Gronwall's inequality, Makedon. Akad. Nauk. Umet. Oddel. Mat.-Tehn. Nauk. Prilozi 13 (1992), no. 2, 23-28.

[9] W. Eckhaus, Asymptotic Analysis of Singular Perturbations, Studies in Mathematics and Its Applications, vol. 9, North-Holland Publishing, Amsterdam, 1979.

[10] A. Erdélyi, W. Magnus, F. Oberhettinger, and F. G. Tricomi, Higher Transcendental Functions. Vol. III, McGraw-Hill, New York, 1955.

[11] R. Gorenflo and S. Vessella, Abel Integral Equations. Analysis and Applications, Lecture Notes in Mathematics, vol. 1461, Springer-Verlag, Berlin, 1991.

[12] G. Gripenberg, S.-O. Londen, and O. Staffans, Volterra Integral and Functional Equations, Encyclopedia of Mathematics and Its Applications, vol. 34, Cambridge University Press, Cambridge, 1990.

[13] J.-P. Kauthen, A survey of singularly perturbed Volterra equations, Appl. Numer. Math. 24 (1997), no. 2-3, 95-114.

[14] P. Linz, Analytical and Numerical Methods for Volterra Equations, SIAM Studies in Applied Mathematics, vol. 7, Society for Industrial and Applied Mathematics, Philadelphia, 1985.

[15] R. B. Paris and D. Kaminski, Asymptotics and Mellin-Barnes Integrals, Encyclopedia of Mathematics and Its Applications, vol. 85, Cambridge University Press, Cambridge, 2001.

[16] I. Podlubny, Fractional Differential Equations, Mathematics in Science and Engineering, vol. 198, Academic Press, San Diego, 1999. UK

Angelina M. Bijura: The New York University in London, 6 Bedford Square, London WC1B 3RA, E-mail address: mbijura@yahoo.com 


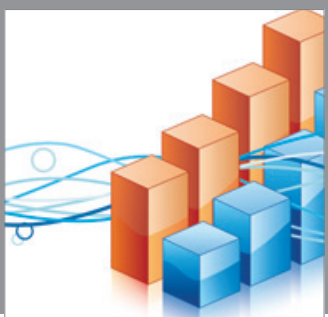

Advances in

Operations Research

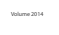

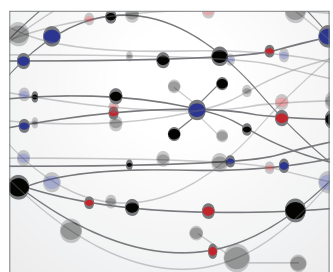

\section{The Scientific} World Journal
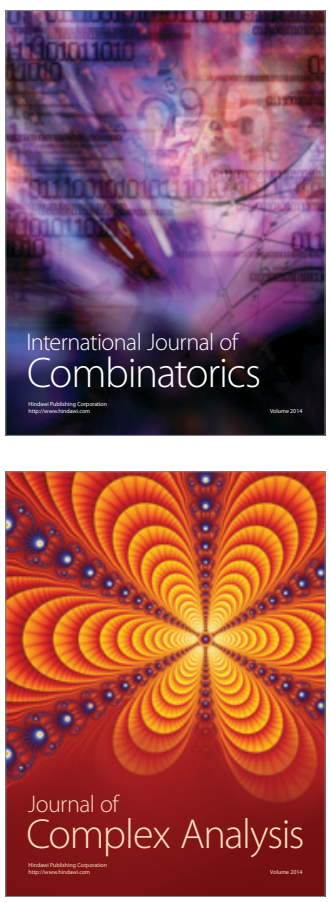

International Journal of

Mathematics and

Mathematical

Sciences
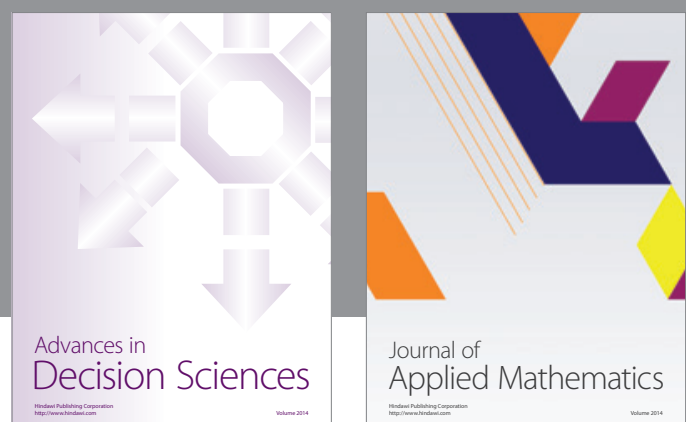

Journal of

Applied Mathematics
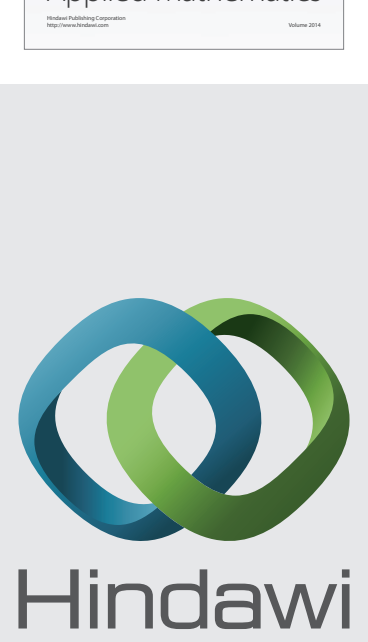

Submit your manuscripts at http://www.hindawi.com
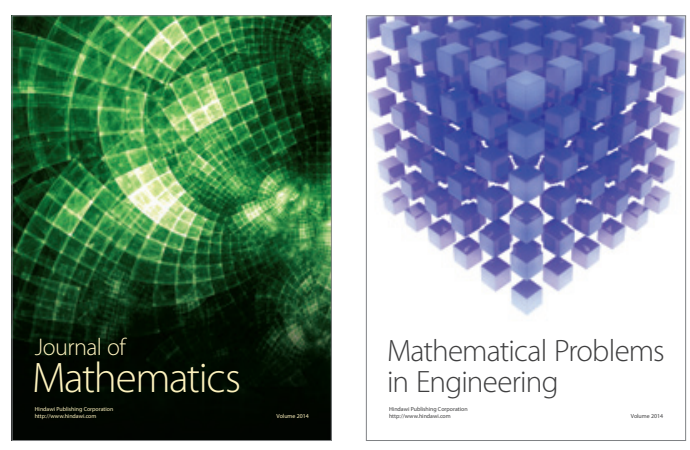

Mathematical Problems in Engineering
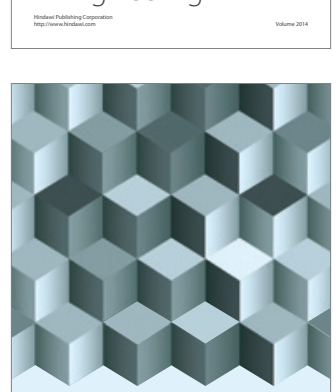

Journal of

Function Spaces
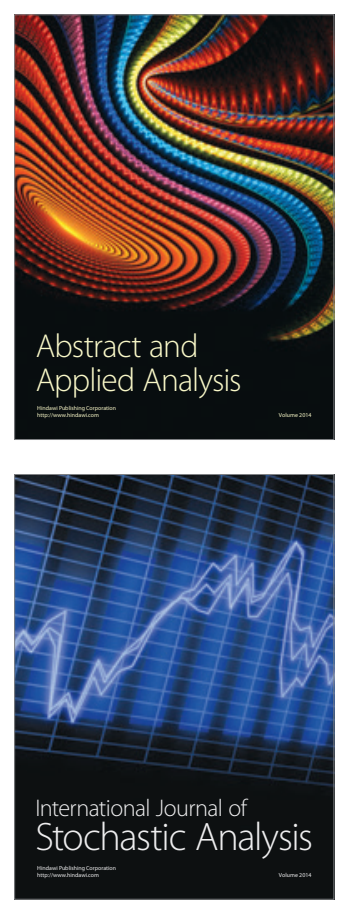

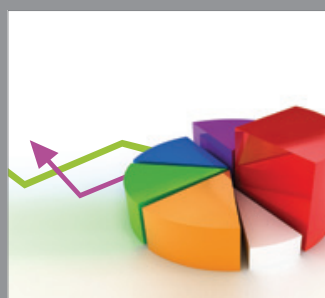

ournal of

Probability and Statistics

Promensencen
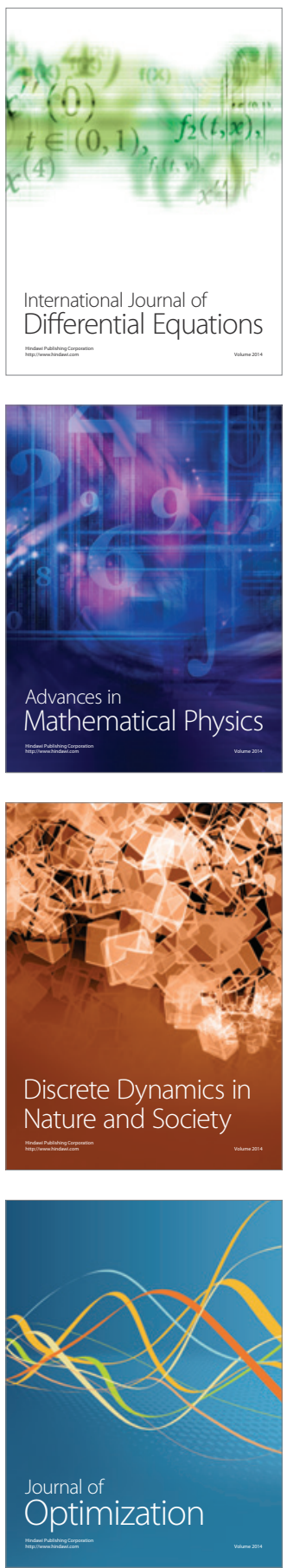